
allemande

47-1| 2015

L'Allemagne et l'Europe | La déprise de l'Empire napoléonien en Allemagne en 1813

\title{
L'Allemagne et la puissance en Europe
}

\section{Christian Lequesne}

\section{(2) OpenEdition \\ 1 Journals}

\section{Édition électronique}

URL : https://journals.openedition.org/allemagne/446

DOI : 10.4000/allemagne.446

ISSN : 2605-7913

Éditeur

Société d'études allemandes

\section{Édition imprimée}

Date de publication : 26 juin 2015

Pagination : 5-13

ISSN : 0035-0974

\section{Référence électronique}

Christian Lequesne, "L'Allemagne et la puissance en Europe », Revue d'Allemagne et des pays de langue allemande [En ligne], 47-1 | 2015, mis en ligne le 13 décembre 2017, consulté le 23 mai 2021. URL http://journals.openedition.org/allemagne/446; DOI : https://doi.org/10.4000/allemagne.446 


\section{L’Allemagne et la puissance en Europe}

\section{- Christian Lequesne*}

En 2012, le sociologue allemand Ulrich Beck publiait un pamphlet intitulé Das Deutsche Europa $a^{(1)}$. Essai à charge d'un intellectuel social-démocrate contre Angela Merkel, l'ouvrage d'Ulrich Beck consiste à affirmer, dans le contexte de la crise économique de 2008, que l'Allemagne fuit ses responsabilités européennes que lui avait assignées Thomas Mann dans son célèbre discours de 1953 à l'université de Hambourg, pour imposer son modèle à l'Europe. Si le livre d'Ulrich Beck est bien trop polémique pour être vraiment convaincant, il est vrai que l'Allemagne réunifiée connaît une renaissance de sa puissance depuis la réunification dont elle ne sait pas toujours que faire. Dans un monde global où l'économie devient un attribut essentiel, l'Allemagne peut être décrite comme la puissance de facto de l'Europe, ce qui lui confère de nouvelles responsabilités qu'elle doit assumer. Pour autant, l'Allemagne n'a pas renoncé à certaines restrictions (notamment militaires) que lui confère son histoire, ce qui fait d'elle une puissance particulière en Europe.

\section{L'Allemagne référentiel du débat français}

Que ce soit une référence positive ou négative, l'Allemagne est présente dans le débat politique des autres États de l'Union européenne. Le cas de la France est à cet égard emblématique. Pour une partie de la droite française, l'Allemagne incarne le pays qui a réussi la réforme structurelle de son économie après avoir été au début des années 2000 "l'enfant malade » de l'Europe; grâce à cela, elle a pu asseoir son leadership au sein de l'Union européenne. À gauche, les socio-démocrates français ont une admiration pour l'économie allemande sans pouvoir trop le dire, car la gauche du Parti socialiste voit dans l'Allemagne un hégémon économique qui mène l'Europe à la fin de l'État providence. C’est au sein de la gauche française que l'on observe,

* Professeur à Sciences Po Paris (CERI).

1 Ulrich BЕск, Das deutsche Europa: neue Machtlandschaften im Zeichen der Krise, Berlin, Suhrkamp, 2012 . 
dans la décennie actuelle, les manifestations les plus explicites d'un anti-germanisme des élites politiques. Dès novembre 2011, le futur ministre du Redressement productif Arnaud Montebourg n'avait ainsi pas hésité à comparer la politique d'Angela Merkel à celle de Bismarck ${ }^{(2)}$. Évincé du gouvernement en août 2014 par le Premier ministre Manuel Valls, Arnaud Montebourg n'hésite pas à ajouter: "Les électeurs français ont voté pour une politique de gauche et ils ont obtenu le programme de la droite allemande ${ }^{(3)}$. Cela sous-entend que l'Allemagne imposerait à la France l'austérité et le renoncement à l'État providence.

La société française est en général plus sereine à l'égard de l'Allemagne que ne le sont les élites politiques. L'anti-germanisme ordinaire a quasiment disparu des représentations. Un sondage IFOP de janvier 2012 montrait que 63 \% des Français avaient une bonne image de l'Allemagne et $19 \%$ une très bonne image, contre $15 \%$ une assez mauvaise image et $3 \%$ une très mauvaise image. En même temps, ce sondage montrait aussi que $36 \%$ des Français n'avaient jamais mis un pied sur le territoire de l'Allemagne et que $73 \%$ déclaraient ne pas du tout maîtriser la langue allemande ${ }^{(4)}$.

Dans l'ensemble, les Français ressentent encore un certain exotisme lorsqu'ils se rendent en voyage en Allemagne. Les témoignages de vacances demeurent toujours assez romantiques. On y raconte la qualité de l'accueil, souvent - il faut bien le souligner - à Berlin ou au sud de l'Allemagne. Les sexagénaires qui ont accompli leur service militaire dans l'ancienne zone française y retournent effectuer des pèlerinages mémoriels. Le service militaire outre-Rhin fut pour beaucoup d'entre eux la première expérience hors de France. L'effet de socialisation ne fut pas négligeable, même si "leur " Allemagne se résuma souvent au trajet entre les casernes et les gares des villes de stationnement ${ }^{(5)}$.

Les narrations françaises des expériences des enfants qui font un séjour en Allemagne sont souvent aussi très intéressantes. L'une des grandes surprises françaises est que l'Allemagne est un pays où le système scolaire est en général plus permissif qu'en France. Les lycéens français qui se rendent en Allemagne dans le cadre des échanges de l'OFAJ (comme les programmes Voltaire ou Sauzay) témoignent souvent d'une ambiance plus libre au Gymnasium ${ }^{(6)}$. Habitués à penser l'éducation comme un parcours qui doit endurcir - car élitiste -, les élèves et les parents français ne comprennent pas pourquoi les Allemands ont au final une économie qui fonctionne si bien avec un système scolaire qui exige moins d'efforts et de concurrence de la part de leurs adolescents. En matière d'éducation, les Français continuent à croire dans la formation d'une petite élite alors que la formation d'un homme « moyen » est davantage un objectif du système éducatif allemand.

2 Voir Le Figaro, 30 novembre 2011.

3 Voir l'échange avec Thomas Piketty dans Les Inrockuptibles, 9 septembre 2014.

4 IFOP, L'image de l'Allemagne en Europe, janvier 2012 (sondage commandé par l'Ambassade d'Allemagne à Paris).

5 On pourra relire Suzie Guth, Les Forces Françaises en Allemagne. La Citadelle Utopique, Paris, L'Harmattan, 1991.

6 Voir les témoignages d'élèves français qui ont accompli le programme Voltaire sur le site de l'OFAJ, http://www.ofaj.org/programme-voltaire. 


\section{Les précautions de la puissance « de facto »}

À l'exception de quelques forces politiques nationalistes (notamment dans les pays d'Europe centrale), il y a en Europe un respect pour la démocratie allemande qui a su tirer des leçons positives de son passé totalitaire en refondant la République fédérale en 1949, puis en opérant une réunification pacifique en 1990. Les Allemands de l'Ouest après 1945 ont fait preuve d'une résilience qui montre une capacité à mobiliser une morale collective. Les Allemands de l'Est n’ont pas été invités à le faire puisque le communisme était censé effacer tout passé nazi. La résilience n'excusera jamais Auschwitz, mais est une leçon d'espérance pour souligner la capacité de n'importe quel peuple à sortir de l'horreur totalitaire pour retrouver la démocratie. Cela n'a pas été facile pour la génération d'après-guerre. Un ancien directeur du Goethe-Institut, qui a servi cette institution dans le monde entier pendant quarante ans, avouait récemment que "ça ne lui a pas été facile d'être Allemand dans bien des pays " ${ }^{(7)}$. Bien entendu, la culpabilité est moins marquée dans la jeune génération qu'elle ne le fut chez les parents et les grands-parents. En même temps, on sent toujours dans la société allemande une conscience du passé totalitaire, surtout que ce passé est maintenant double (national-socialiste et communiste pour l'ex-RDA). Est-ce un poids inutile comme l'avait pensé il y a une quinzaine d'années le romancier Martin Walser ${ }^{(8)}$ ? On peut objecter le contraire: il s'agit d'une ressource morale. Être conscient de son passé totalitaire constitue une force de la conscience démocratique d'un pays. Dans le cas de l'Allemagne, elle explique peut-être l'absence dans le paysage politique d'une extrême droite structurée, alors que la plupart des autres pays européens, y compris la France, disposent de tels partis extrémistes.

Une deuxième caractéristique de l'Allemagne en Europe est la capacité de sa société à affronter les situations difficiles en construisant du consensus. Plus encore que la culture industrielle, la fabrication de consensus est un facteur déterminant de la sortie de crise économique des années 2010 en Allemagne. La formation à deux reprises d'une grande coalition entre la CDU/CSU et le SPD (entre 2005 et 2009, puis depuis 2013) en est la manifestation. L'aptitude à s'entendre entre les partis politiques, les chambres de commerce, les industries et les syndicats est porteuse d'une modernité politique pour l'Europe. On est là dans une tradition très différente de la France qui vit encore ce que l'historienne Mona Ozouf appelle un «surmoi révolutionnaire ${ }^{(9)}$ selon lequel le pays ne peut avancer que dans l'affrontement violent entre deux camps. Il faut ajouter une autre différence notable: une faible croyance en Allemagne à l'enchantement de la politique. Dans le cas français, l'historien Charles Renouvier avait, à la fin du XIX ${ }^{e}$ siècle, déjà remarqué ce trait de société lorsqu'il affirmait: «En France, tout ce qui n’est pas idéal est misère » ${ }^{(10)}$. L'attitude modeste d'Angela Merkel qui, réélue pour la deuxième fois très confortablement, le 17 décembre 2013, déclare à ses électeurs que c'est maintenant que les choses difficiles commencent, n'existerait pas de la même manière en

7 Conversation avec l'auteur, 6 septembre 2014

8 On relira le discours de Walser du 12 octobre 1998 prononcé à l'occasion de la remise du prix des Libraires allemands à Francfort.

9 Voir l'émission des Matins de France Culture (invitée Mona Ozouf) du 7 avril 2014.

10 Ibid. 
France $^{(11)}$. La rhétorique politique française voudrait qu'un vainqueur d'une élection souligne l'espoir d'un nouvel avenir idéal. La culture du consensus et le renoncement à l'idéalisme (pourtant composante essentielle de la philosophie allemande du XVIII siècle) sont des clés d'une modernité allemande dans l'Europe de 2014.

Bien que l'économie allemande ne soit pas sans faille (démographie déclinante, emplois précaires), les bons indicateurs macro-économiques (un budget à l'équilibre en 2014) et le dynamisme des exportations (un excédent commercial de 22,2 milliards d'euros en juillet 2014) contribuent à conférer au pays le statut de puissance en Europe et dans le monde. L'Allemagne est devenue la puissance de facto de l'Europe, ce que les Allemands ont eux-mêmes parfois du mal à assumer, tant ils se méfient de leur propre hégémonie depuis 1945. Il convient cependant d'évoquer deux limites de la réussite allemande et un manque.

La première limite est liée à cette idée répandue chez un certain nombre d'élus mais aussi de citoyens allemands selon laquelle les pays de l'Europe, s'ils veulent progresser dans leur réforme, doivent imiter le modèle allemand. Si les réformes allemandes sont une source d'inspiration pour les autres Européens dans le cadre d'un processus plus général «d'européanisation » des politiques publiques, elles ne peuvent pas être appliquées exactement de la même manière partout ailleurs. Chaque pays européen connaît sa propre trajectoire historique (ce que Max Weber appelait l'historicité et les institutionnalistes américains la path dependency $\left.{ }^{(12)}\right)$ qui ne rend jamais les conditions de réception d'une politique publique identiques d'un territoire à un autre. Pour revenir à la France, la difficulté à trouver du consensus politique, évoqué plus haut, provient d'une histoire singulière. Aucun gouvernement français - de gauche ou de droite - ne peut l'effacer d'un trait de plume du jour au lendemain pour créer les conditions d'une grande Mitbestimmung à l'allemande. La montée du parti Alternative für Deutschland (AfD), qui a fait 7,1\% des voix aux élections européennes de juin 2014 et entre 9,7 \% et $12,2 \%$ aux élections régionales de Saxe, Thuringe et Brandebourg, montre qu'une catégorie d'électeurs allemands rejette une Europe dont les États n'auraient pas tous la même propension à réformer l'économie que l'Allemagne. Si l'AfD ne peut pas être classé comme un parti d'extrême droite, il reflète en tout cas l'enfermement d'un électorat, dont il faut connaître un peu plus la composition sociologique ${ }^{(13)}$, sur un modèle économique réputé exemplaire.

Liée à la première, la deuxième limite consiste à opposer d'une manière un peu trop caricaturale une Europe du Nord vertueuse et travailleuse, qui aurait fait sienne le célèbre adage souabe "schaffe, schaffe und Häusle baue ", à une Europe du Sud dispendieuse et paresseuse. Cette opposition nourrit un populisme en Allemagne au-delà même de l'électorat $\mathrm{AfD}$, comme en a attesté une campagne de presse très

11 Discours disponible sur http://www.lepoint.fr/video/video-extrait-du-discours-d-angela-merkel22-09-2013-1733999_738.php.

12 Voir Paul Pierson, Politics in Time: History, Institutions and Social Analysis, Princeton, Princeton University Press, 2004.

13 Voir Olaf Sundermeyer, "Alternative für Demagogen », RBB online, 4 juin 2014, http://www.rbbonline.de/politik/beitrag/2014/06/Mitte-Studie-ueber-rechtsextreme-Einstellung-DeutschlandBrandenburg-AfD.html. 
dure (choquante même dans le cas du tabloïd Bild-Zeitung ${ }^{(14)}$ ) au début de la crise grecque. Ce populisme des riches, opposés à tout transfert fiscal vers des territoires moins favorisés, est une évolution plus générale en Europe. On le retrouve en Italie dans le discours de la Ligue du Nord ou en Espagne dans celui de certains indépendantistes catalans. L'Allemagne, qui doit beaucoup à l'Europe pour ce qui est de sa démocratisation après 1945, mais aussi pour l'exportation de ses produits industriels, connaît un mouvement contre la solidarité budgétaire en Europe. Cela ne veut pas dire que les gouvernements allemands ont arrêté les transferts budgétaires vers les autres pays européens (l'Allemagne reste le premier contributeur net au budget de l'Union européenne avec 301 euros par habitant en 2012) mais que de plus en plus de citoyens trouvent ces transferts de moins en moins légitimes.

Le manque concerne l'engagement de l'Allemagne dans la sécurité du monde. Bien entendu, si les Allemands sont obligés de comprendre les trajectoires historiques des autres Européens, la réciprocité s'applique. Les hésitations allemandes à être plus ambitieux dans le domaine militaire sont en effet le résultat direct de l'histoire allemande $\mathrm{du} \mathrm{xx}^{\mathrm{e}}$ siècle. La participation allemande à la sécurité du monde pourrait cependant être supérieure à ce qu'elle est actuellement. Pour se limiter au produit intérieur brut consacré à la défense, l'Allemagne y consacre à peine 1,3\% contre 2,2\% pour la France $^{(15)}$. En 2011, l'Allemagne a refusé d'intervenir aux côtés des Britanniques et des Français en Libye, afin d'aider l'opposition à en finir avec le régime de Kadhafi. On a beaucoup expliqué ce choix comme celui du ministre libéral des Affaires étrangères, Guido Westerwelle, qui voulait ainsi ménager aux élections régionales l'électorat du FDP opposé aux interventions militaires. En fait, il n'en fut rien car le FDP n'arriva pas à franchir la barre des $5 \%$ qui lui aurait permis de continuer à disposer de députés au Landtage de Bade-Wurtemberg, de Saxe-Anhalt et de Sarre. Par ailleurs, la chancelière Merkel, CDU, ne s'est pas opposée frontalement à la position exprimée par le vice-chancelier, ministre des Affaires étrangères. Le scénario est assez identique pour l'opération militaire visant à stopper la progression des groupes djihadistes au Mali en 2013: l'Allemagne n'est pas intervenue aux côtés de la France. Les réactions internationales à l'égard d'une Allemagne qui se comporterait comme « une grande Suisse » ont été assez défavorables ${ }^{(16)}$.

Cette situation explique d'ailleurs qu'il y ait un débat en Allemagne autour d'une plus grande ambition militaire. Si l'Allemagne depuis 1990 a déjà beaucoup évolué en matière de présence militaire internationale, comme le montre la présence de troupes dans les ex-Républiques yougoslaves ou en Afghanistan, il s'agirait d'être plus engagé dans les «frappes chirurgicales ». La nécessité d'une plus grande ambition pour la Bundeswehr en matière d'intervention extérieure a été exprimée par le président fédéral Joachim Gauck, mais aussi par le ministre SPD des Affaires étrangères FrankWalter Steinmeier et la ministre CDU de la Défense Ursula von der Leyen lors de la

14 Voir en particulier la première de couverture du 27 octobre 2010, « Verkauft doch eure Inseln, ihr Pleite-Griechen ».

15 http://donnees.banquemondiale.org/indicateur/MS.MIL.XPND.GD.ZS.

16 Timothy Garton Ash, "France plays hawk, Germany demurs. Libya has exposed Europe's fault lines », The Guardian, 24.03.2011. 
Wehrkunde de Munich en janvier $2004^{(17)}$. Le point intéressant est qu'il n'y a pas de réel soutien de la société allemande à l'égard de cet engagement militaire accru. Un sondage de 2014 montre que seulement $37 \%$ des Allemands considèrent prioritaire une plus grande présence militaire de leur pays dans le monde ${ }^{(18)}$. L'après-Seconde Guerre mondiale a ancré profondément l'Allemagne dans une tradition diplomatique $\mathrm{du}$ « soft power » qui pose un problème à l'Europe dès lors que les budgets militaires sont en diminution partout et que la Grande-Bretagne n'est pas en mesure d'assumer - pour des raisons de politique intérieure - tout pas supplémentaire vers une Europe de la défense.

\section{Perspectives européennes}

La contribution de l'Allemagne à la construction européenne a été essentielle depuis les années cinquante ${ }^{(19)}$. Si l'on réfléchit en termes d'idéal type, la contribution de l'Allemagne à la construction européenne s'est traduite sur la longue période par un engagement en faveur de la méthode décisionnelle communautaire: celle qui favorise le rôle de la Commission, du Parlement européen et des instruments de législation européenne contraignants (règlement ou directive). À l'inverse, les Britanniques ont plutôt toujours cherché à défendre la méthode intergouvernementale et les Français se sont souvent situés dans un entre-deux, visant à défendre la méthode communautaire pour l'intégration des marchés mais la méthode intergouvernementale pour la politique étrangère commune et la défense ${ }^{(20)}$.

L'engagement de l'Allemagne en faveur de la méthode communautaire a permis de trouver des compromis avec la France qui ont favorisé les grandes avancées de la construction européenne. Pour ne prendre que deux exemples, ce fut le cas pour le marché intérieur en 1986 et l'Union économique et monétaire en 1992. Depuis l'arrivée à la chancellerie d'Angela Merkel, l'Allemagne - comme tous les pays de l'Union européenne - connaît un infléchissement de ses préférences institutionnelles vers l'intergouvernementalisme qui s'est traduit par exemple par la signature en mars 2012 du traité sur la stabilité, la coordination et la gouvernance en Europe. L'intergouvernementalisme est la méthode qui permet aux responsables politiques européens d'affirmer à des opinions publiques dans le doute qu'ils n'ont pas renoncé à défendre la souveraineté nationale. C'est ainsi que l'on doit lire la proposition d'Angela Merkel, dans son discours au Collège de Bruges de novembre 2010. Elle y parle du besoin pour l'Union européenne de développer une "neue Unionsmethode " ${ }^{(21)}$ qui doit s'imposer sur la recherche systématique de solutions communautaires dès lors qu'elle permet de produire des résultats. Dans ses arrêts récents, la Cour constitutionnelle fédérale

17 Les trois discours sont disponibles en allemand sur le site https:/www.securityconference.de/ veranstaltungen/munich-security-conference/msc-2014/reden/.

18 Chiffre cité par Frédéric Lemaître dans Le Monde du 2.10.2014.

19 Simon Bulmer, "Germany in Europe: from 'tamed power' to normalized power », International Affairs, 86/5 (2010), p. 1051-1073.

20 Ulrich Krotz et Joachim Schild, Shaping Europe: France, Germany and the Embedded Bilateralism from the Élysée Treaty to the 21th Century, Oxford, Oxford University Press, 2012.

21 Pour le texte complet du discours d'Angela Merkel, voir http://www.bundesregierung.de/Content/ DE/Bulletin/2010/11/111-1-bk-akadamisches-jahr.html. 
a aussi souligné de manière récurrente l'importance de saisir le Bundestag, ce qui est une autre manière de rappeler que les institutions d'État doivent rester au cœur du processus de décision européen ${ }^{(22)}$.

À ce propos, les diplomates britanniques - généralement opposés à la sortie complète de leur pays de l'Union - aiment rappeler que le gouvernement allemand fera tout pour que la Grande-Bretagne négocie un statut qui lui convienne. L'idée sous-jacente est que l'Allemagne a besoin de la Grande-Bretagne, généralement libérale sur le plan de la doctrine économique, pour prendre part aux décisions relatives aux questions de marché intérieur. Ce débat soulève la question d'une alternative au retrait pur et simple de la Grande-Bretagne, prévu par l'article 50 du traité sur l'Union européenne, en cas de référendum négatif en 2017. L'hypothèse d'une " adhésion différenciée ", qui irait au-delà de la seule participation à l'Espace économique européen, sera reconsidérée par les experts sans que les réponses paraissent très simples à élaborer sur le plan politique et juridique ${ }^{(23)}$.

Les difficultés économiques de l'Europe mettent les économistes face à des scénarios différents depuis 2008. C’est une excellente démonstration, au demeurant, que l'utilisation de quelques modèles issus de la physique n'a pas transformé l'économie en une science exacte, contrairement à ce que s'autorisent à penser certains économistes. Aucun expert ne prouvera donc de manière scientifique que le modèle allemand d'équilibre des finances publiques est le "seul » qui permette aux économies européennes de retrouver la croissance. Il se trouve cependant que ce modèle d'équilibre des finances publiques est, en 2014, celui qui fournit empiriquement les meilleurs résultats. L'Allemagne, qui était l'enfant malade de l'économie européenne au début des années 2000, a réussi ses réformes en appliquant ce modèle et en tire une forte légitimité en Europe. Le décrochage entre l'économie allemande et l'économie française depuis 2008 est un problème politique pour l'Union européenne.

En Allemagne, les responsables politiques et les économistes (profession qui jouit d'un prestige et d'une écoute) ont souvent tendance à considérer la Sparpolitik comme une vérité scientifique parce qu'elle produit des effets chez eux à court terme. Mais les risques de déflation et de récession, liés à une politique de l'offre trop restrictive, que l'on souligne dans d'autres pays de l'Union, ne sont pas des arguments totalement insensés. L'Allemagne souffre d'un manque d'investissements comme la plupart des pays européens. Mario Draghi, le président de la Banque centrale européenne qui a récemment desserré les taux d'intérêt, semble être sensible à la nuance, ce qui lui vaut une relation difficile avec Jens Weidmann, le président de la Bundesbank garant d'une orthodoxie budgétaire stricte ${ }^{(24)}$. Le gouvernement d'Angela Merkel, qui a atteint en 2014 l'équilibre budgétaire dans les finances publiques, alors que la France affiche un déficit de $4,4 \%$ du PIB, ne semble pas cependant vouloir s'écarter du modèle de la rigueur, qui par ailleurs ne déplaît pas complètement non plus au SPD. Il est assez certain, dans un climat de

22 Voir en particulier l'arrêt de la Cour constitutionnelle fédérale sur le Mécanisme européen de stabilité de septembre 2012.

23 Voir " EU Brexit: Looking at the case for an exit ", Investec Economics, 23.03.2014, https://www. investec.com/content/dam/investec/investec-international/documents/Philip\%20Shaw\%20\%20 Week\%20Ahead\%20PDFs/ECOU_91720.pdf.

Voir Les Échos, 5.09.2014. 
montée de l'AfD, que ce thème restera une source de conflits avec d'autres gouvernements européens, en particulier la France, au sein de l'Union européenne. L'incapacité française a démontré concrètement qu'une politique keynésienne plus dépensière qui produit des résultats positifs pour sa propre économie est perçue cependant comme une faiblesse objective. Des pays comme la Lettonie ou la Pologne, qui ont réalisé des coupes budgétaires strictes et retrouvent une meilleure croissance, se sentent attirés davantage par le modèle allemand de la Sparpolitik que par le modèle français. Il en découle des conséquences pour le leadership politique de l'Allemagne et pour le rôle de la relation franco-allemande au sein de l'Union européenne.

Une dernière perspective concerne l'attitude de l'Allemagne à l'égard de la Russie au sein de la politique étrangère et de sécurité de l'Union européenne. Vladimir Poutine mène une politique de reconquête de l'honneur national russe qui passe par une diplomatie du rapport de force à l'égard de son voisinage. L'annexion de la Crimée par la Fédération de Russie, en mars 2014, a été un point de rupture avec l'Union européenne, tout comme l'a été le lancement du missile, dans l'est de l'Ukraine, qui a coûté la vie aux passagers de l'avion de la Malaysian Airlines, en juillet 2014. Trois vagues de sanctions européennes ont été adoptées par l'Union européenne contre la Russie depuis l'annexion de la Crimée. En règle générale, le lancement en 2004 par l’Union européenne, sur proposition de la Pologne et de la Suède, d'une politique européenne de voisinage à l'égard de l'Ukraine, de la Moldavie et du Caucase a conduit Moscou à voir Bruxelles davantage comme une entité politique et donc comme un concurrent gênant ${ }^{(25)}$. Le président russe Poutine utilise contre l'Union européenne deux registres: d'une part, la menace de couper les approvisionnements d'hydrocarbures; d'autre part, l'exploitation de toute contradiction entre les diplomaties des États de l'Union européenne à son égard. L'Allemagne, qui dépend à $37 \%$ de la Russie pour ses approvisionnements en gaz, s'est ralliée aux sanctions suite à l'invasion de la Crimée, tout comme la France et la Grande-Bretagne. Il existe néanmoins dans les forces politiques allemandes une volonté de ménager la Russie qui procède au moins de trois fondements: le poids de l'histoire, l'économie (la dépendance énergétique mais aussi les investissements des entreprises allemandes en Russie) et, enfin, une représentation géopolitique de l'Europe qui se doit de prendre en compte le facteur russe. Le discours de ménagement de la Russie se trouve dans tous les partis politiques. Il est particulièrement présent au sein de la social-démocratie dont l'un des représentants, FrankWalter Steinmeier, détient en 2014 le portefeuille des affaires étrangères. Le relatif accommodement dont fait preuve la diplomatie allemande à l'égard de la Russie tend à raidir la relation entre Paris et Varsovie qui s'est pourtant considérablement assouplie depuis l'élection en 2007 de Donald Tusk au poste de Premier ministre de la Pologne. Les Polonais souhaiteraient que l'Allemagne ait une position plus ferme à l'égard de la Russie, car le passé communiste leur donne l'impression d'être les garants moraux de la liberté et de «l'européanisation » des Ukrainiens, des Moldaves ou encore des Géorgiens. L'oscillation allemande à l'égard de la Russie de Poutine entre condamnation de la diplomatie du bâton et préservation des intérêts économiques est un élément de

25 Voir Florent Parmentier, Les chemins de l'État de Droit: la voie étroite entre Europe et Russie, Paris, Presses de Sciences Po, 2014. 
rapprochement avec la diplomatie française. Comme Berlin, Paris ne sait pas vraiment comment réagir à l'égard de Poutine. Le gouvernement Valls se trouve ainsi confronté au projet de vente de deux navires de guerre Mistral aux Russes, qui avait été initié par le président Sarkozy. Il s'agit de ventes portant sur 1,2 milliard d'euros que le gouvernement français a gelées en 2014 , mais sans y renoncer définitivement ${ }^{(26)}$. Plus encore que pour la France et la Grande-Bretagne, l'orientation de l'industrie allemande vers l'exportation, les besoins de ressources naturelles et la tradition d'une diplomatie de " soft power » encadrent la marge de manœuvre de l'Allemagne à l'égard de Moscou. Mais il ne serait pas juste pour autant de ne fournir que cette explication. Il existe aussi chez beaucoup d'hommes politiques allemands une représentation sociale issue de l'histoire selon laquelle déstabiliser la Russie est de mauvais augure non seulement pour l'Allemagne mais aussi pour l'Europe dans son ensemble ${ }^{(27)}$. La définition d'une politique étrangère et de sécurité commune à l'égard de la Russie ne peut dès lors être appréhendée que comme une perspective difficile.

L'Allemagne est sans aucun doute une puissance en Europe. Parfois l'Allemagne elle-même a du mal à s'en convaincre. Pour autant, l'Allemagne n'est pas un État qui se comporte comme une puissance sans retenue. Profondément démocratique, sa société reste marquée par son histoire $\mathrm{du} \mathrm{xx}^{\mathrm{e}}$ siècle, et notamment par sa responsabilité à l'égard du national-socialisme. L'idée selon laquelle l'histoire ne compterait plus en Allemagne n'est pas juste, pas plus que ne l'est l'image d'une Allemagne qui resterait l'État semi-souverain d'avant la réunification. L'Allemagne est une puissance spécifique en Europe qu'il faut étudier davantage pour alimenter la réflexion comparative sur le statut de la puissance étatique au $\mathrm{xxI}^{\mathrm{e}}$ siècle.

\title{
Résumé
}

L'Allemagne réunifiée connaît une renaissance de sa puissance en Europe depuis 1989, dont elle ne sait pas toujours que faire. Dans un monde globalisé où l'économie devient un attribut essentiel, l'Allemagne peut être décrite comme la puissance de facto de l'Europe, ce qui lui confère de nouvelles responsabilités qu'elle doit assumer. Pour autant, l'Allemagne n'a pas renoncé à certaines restrictions (notamment militaires) que lui impose son histoire, ce qui fait d'elle une puissance particulière en Europe.

\begin{abstract}
Reunified Germany is experienced a revival of its power in Europe since 1989. Germany does not always know how to behave with this power revival. In a globalized world, where economy has become a major attribute of power, Germany can be described as the de facto power of Europe, with an obligation to fulfil new responsibilities. However, Germany has not renounced to a certain number of limits (for instance in the military field) coming from its history. It makes Germany still a special country regarding the exercise of power in Europe.
\end{abstract}

26 «Mistral: quelles conséquences après la suspension de la vente aux Russes? », Le Monde, 4.09.2014.

27 Andrea Kamp, Peter Jahn, Philip Springer, Unsere Russen, unsere Deutschen: Bilder vom Anderen 1800 bis 2000, Berlin, Ch. Links Verlag, 2007. 